\title{
R\&D ERL: G5 test and Commissioning Plan
}

\author{
E. Pozdeyev and D. Kayran
}

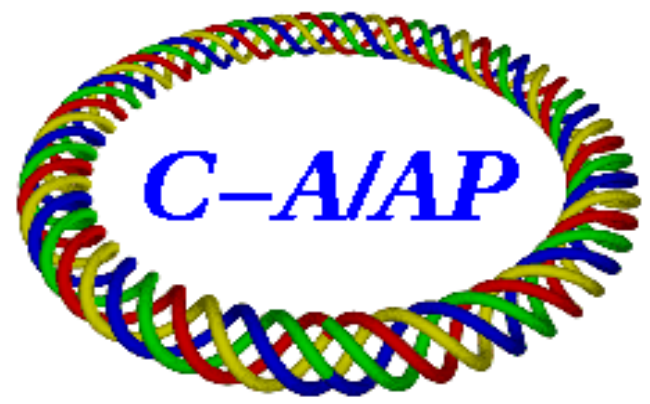

\section{Collider-Accelerator Department Brookhaven National Laboratory Upton, NY 11973}

Notice: This document has been authorized by employees of Brookhaven Science Associates, LLC under Contract No. DE-AC02-98CH10886 with the U.S. Department of Energy. The United States Government retains a nonexclusive, paid-up, irrevocable, world-wide license to publish or reproduce the published form of this document, or allow others to do so, for United States Government purposes. 


\section{ERL R\&D: G5 test and Commissioning Plan}

Eduard Pozdeyev, Dmitry Kayran BNL, CAD

\section{Table of contents:}

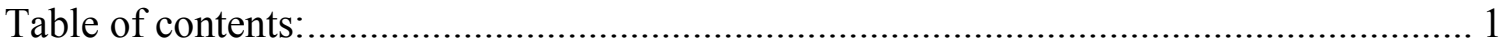

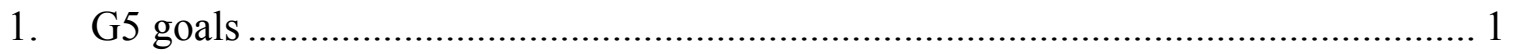

2. G5 setup, its main parameters, and operational principles ................................. 3

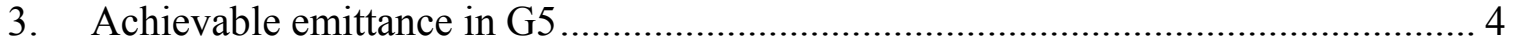

3.1. Optimized projected emittance after 5-cell cavity ........................................ 4

3.2. Optimized projected emittance before 5-cell cavity (at the location of pepper pots) 5

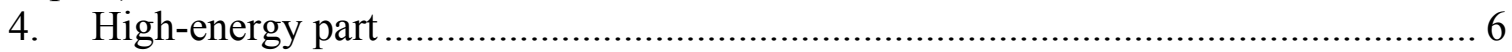

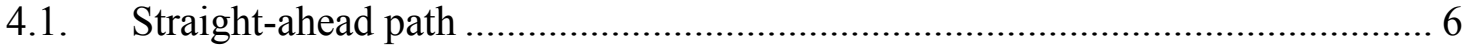

4.2. The bend: energy spread and slice emittance measurements.......................... 8

4.2.1. Slice parameters of the off-crested beam............................................... 9

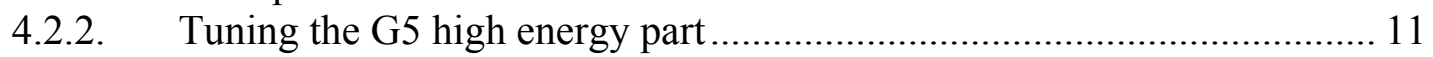

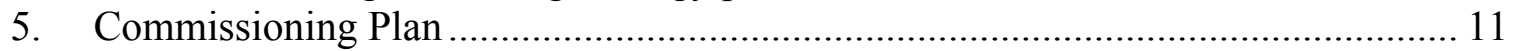

\section{G5 goals}

Gun-to-5-cell cavity (G5) setup (Fig 1-2) can be considered as the first stage of the final BNL ERL design. The goal of the G5 setup is to test critical ERL components with the beam and characterize the beam produced by the gun. Also, this test will be used to assess effectiveness of the zigzag merger, which will be installed later in the ERL setup. The major components under the test will include the SRF gun, the five-cell SRF cavity, vacuum components, parts of the control and diagnostic systems.

G5 is designed to measure the following beam parameters:

- $\quad$ projected bunch emittance $(\varepsilon)$ and Twiss parameters $(\alpha, \beta)$

- slice emittance

- bunch length

- longitudinal and transverse halo 


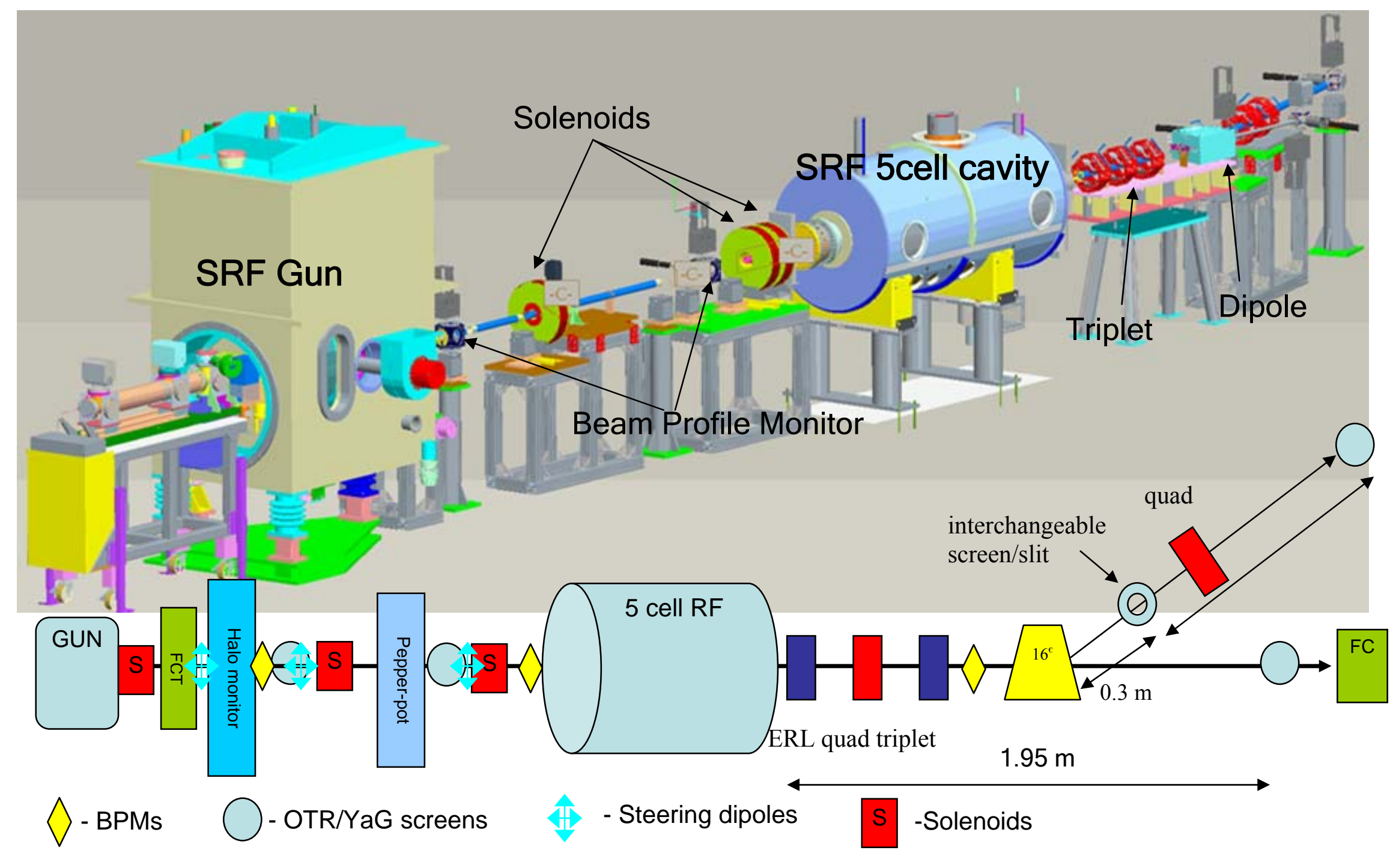

Figure 1: G5 test layout 


\section{G5 setup, its main parameters, and operational principles}

Figure shows the floor plan of the G5 setup. G5 consists of

- SRF gun and a SC solenoid (not shown). The gun is expected to produce bunches with a kinetic energy of $2 \mathrm{MeV}$ at a maximum rep. rate of $10 \mathrm{MHz}$. The charge of the bunches is expected to be between a few tens of $\mathrm{pC}$ 's to up to 5-10 $\mathrm{nC}$.

- Low energy line between the gun and the 5-cell cavity. The line includes a laser port, three solenoids (only two used, the one closer to the cavity is off), two viewers, one in-flange fast current transformer, and halo monitors/scrapers. Also, the line includes two ports reserved for pepper pots. These pepper pots along with one of the viewers can measure the emittance and other beam parameters of the low energy beam.

- 5-cell SRF cavity. The maximum expected energy gain per cavity is approx. 20 $\mathrm{MeV}$. All the simulations presented in this note were done for an energy gain of approx. $15 \mathrm{MeV}$.

- High energy part. The high energy part contains an ERL quad triplet and an ERL dipole. Behind the dipole, the beam goes straight ahead if magnet is off. The straight-ahead line is terminated by a viewer and a low-power dump. This line can be used for a measurement of the total projected emittance. If the magnet is on, the beam trajectory is deflected by $16^{\circ}$. After the bend, there is a diagnostics station containing a vertical slit and a viewer. If the beam is projected on the viewer the beam energy spread can be measured via its horizontal size and the dispersion function. If the slit is inserted instead of the viewer, a longitudinal slice with a given energy is cut out of the beam. This slice is projected on the second viewer at the end of the bent line. The doublet located in the bent line can be used to measure the vertical emittance of the chosen slice.

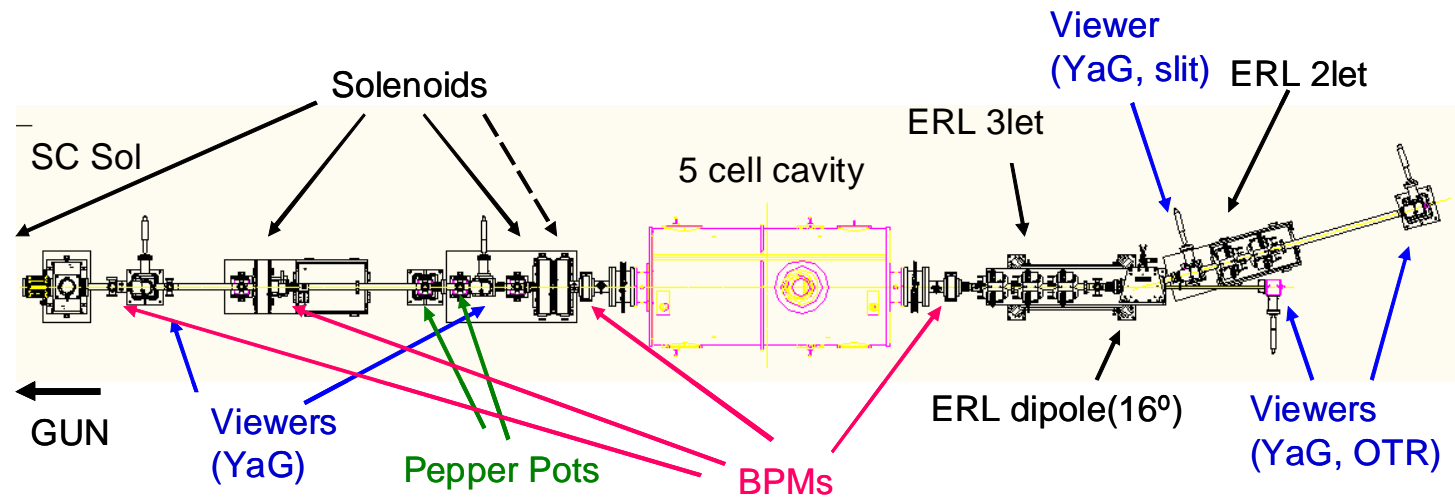

Figure 2: G5 floor plan. The length of the show part of G5 (without the gun) is approx. 9 meters. 


\section{Achievable emittance in $\mathbf{G 5}$}

\subsection{Optimized projected emittance after 5-cell cavity}

An ultimately achievable value of the projected emittance at a high charge per bunch is an important characteristic of a photogun and of a significant interest. Table 1 shows the strength of the solenoids and the optimized normalized projected emittance simulated with PARMELA $25 \mathrm{~cm}$ behind the last flange of the cavity. The emittace is shown for 1.4 $\mathrm{nC}$ and $5 \mathrm{nC}$ "beer can" (BC - initially uniformly charged distribution) and for $1.4 \mathrm{nC}$ distribution with a more realistic simulated laser profile with longer transverse and longitudinal tails. The realistic distribution also included variations of the linear charge density due to an overlap of individual laser pulses. The cathode temperature was $0.4 \mathrm{~K}$.

Table 1: Achievable normalized projected emittance after the 5-cell cavity. The length of hard-edge solenoids was $10 \mathrm{~cm}$.

\begin{tabular}{|l|c|c|c|c|}
\hline $\begin{array}{l}\text { Charge }(\mathrm{nC}) / \\
\text { Dist. }\end{array}$ & $\begin{array}{l}\text { Length(deg. }) / \\
\mathrm{R}_{\max }(\mathrm{mm})\end{array}$ & $\begin{array}{l}\mathrm{E}_{\text {low }} / \mathrm{E}_{\text {high }} \\
(\mathrm{MeV})\end{array}$ & Solenoids $(\mathrm{G})$ & Emittance $(\mu \mathrm{m})$ \\
\hline $1.4 / \mathrm{BC}$ & $12 / 2.5$ & $2 / 16.7$ & $878,412,562$ & $\mathbf{1 . 3}$ \\
\hline $1.4 /$ Realistic & $12 / 3.5$ & $2 / 16.7$ & $885,428,612$ & $\mathbf{2 . 3}$ \\
\hline $5 / \mathrm{BC}$ & $14 / 3.5$ & $2.5 / 17.2$ & $1036,430,660$ & $\mathbf{1 . 7}$ \\
\hline
\end{tabular}

Figure shows the beam size corresponding to the solenoid settings shown in Table 1 . The beam radius reaches aprox. $1 \mathrm{~cm}$ for $1.4 \mathrm{nC}$ bunches and almost $1.5 \mathrm{~cm}$ for $5 \mathrm{nC}$ bunches. The beam pipe radius is approx. 10 up to $190 \mathrm{~cm}$. After $190 \mathrm{~cm}$ the beam pipe radius decreases to $2.4 \mathrm{~cm}$. It is important to note that the beam size can be significantly reduced by increasing the solenoidal focusing. However, this will increase the projected emittance. 


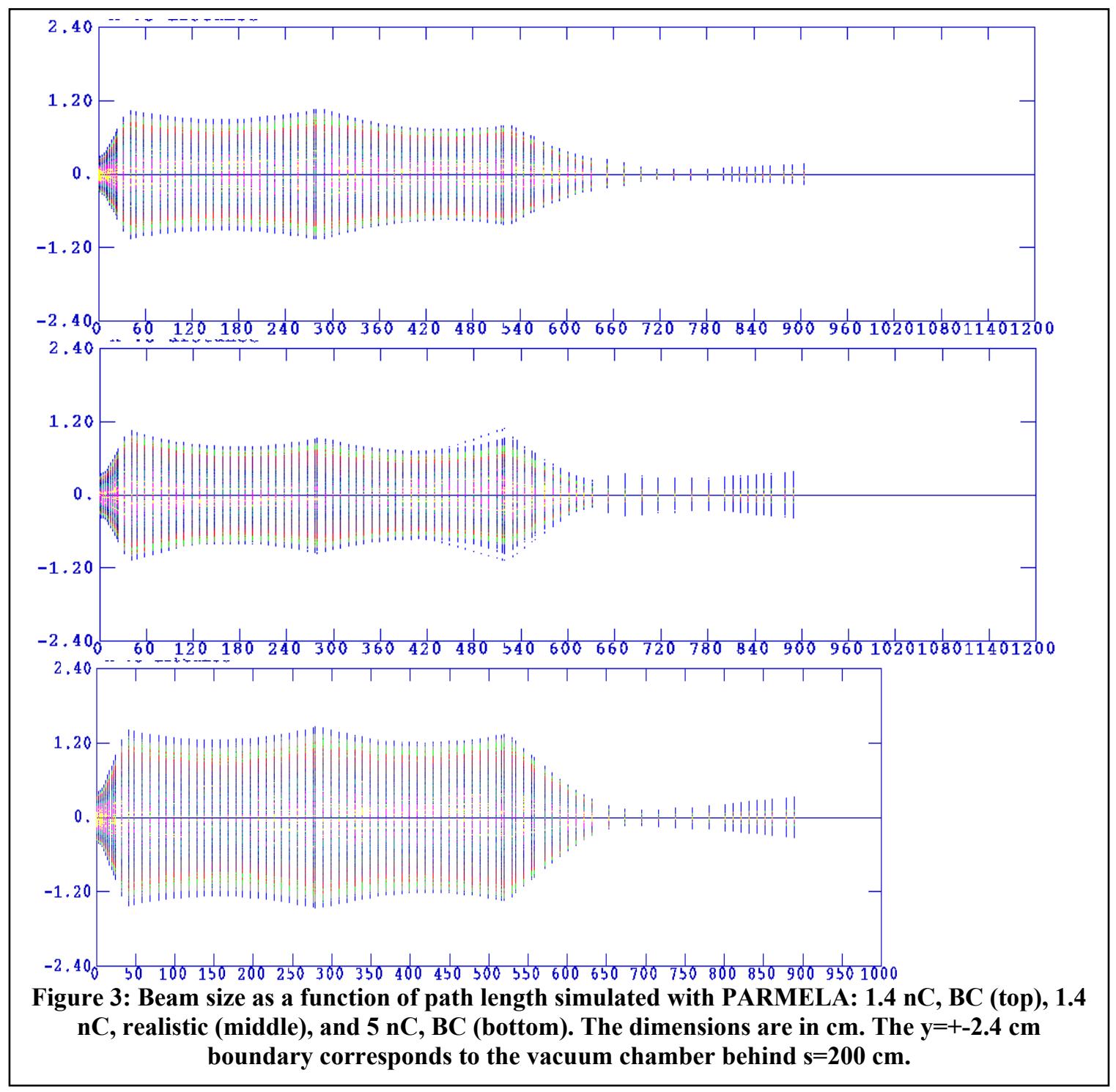

\subsection{Optimized projected emittance before 5-cell cavity (at the location of pepper pots)}

Pepper pots situated in the low energy line have advantages in comparison to quadrupole scans at higher energies: pepper pot measurements do not require running the 5-cell, saving LHe, and can be significantly faster than quads scans. Table 2 shows the optimized emittance at the location of pepper pots simulated by PARMELA.

Table 2: Achievable projected emittance in the low energy line at the location of the pepper pots.

\begin{tabular}{|l|c|c|c|c|c|}
\hline $\begin{array}{l}\text { Charge }(\mathrm{nC}) / \\
\text { Dist. }\end{array}$ & $\begin{array}{l}\text { Length(deg.)/ } \\
\mathrm{R}_{\max }(\mathrm{mm})\end{array}$ & $\begin{array}{l}\mathrm{E}_{\text {low }} \\
(\mathrm{MeV})\end{array}$ & $\begin{array}{l}\text { Pepper pot } \\
\text { (first/second) }\end{array}$ & $\begin{array}{l}\text { Solenoids } \\
(\mathrm{G})\end{array}$ & Emittance $(\mu \mathrm{m})$ \\
\hline $1.4 / \mathrm{BC}$ & $12 / 2.5$ & 2 & 1 & 919,618 & 1.2 \\
\hline 1.4 / Realistic & $12 / 3.5$ & 2 & 2 & 860,556 & 2.6 \\
\hline $3.7 /$ Realistic & $14 / 35$ & 2.5 & 2 & 1054,653 & 3.8 \\
\hline 5 / BC & $14 / 3.5$ & 2.5 & 1 & 1088,713 & 2.2 \\
\hline
\end{tabular}




\section{High-energy part}

The high energy part follows the 5-cell cavity and consists of an ERL triplet, an ERL dipole deflecting the beam by $16^{\circ}$, an ERL doublet, two viewers with interchangeable $\mathrm{YaG}$ and OTR screens, and one more viewer with a slit instead of the OTR screen. Figure shows the layout of the high energy part.

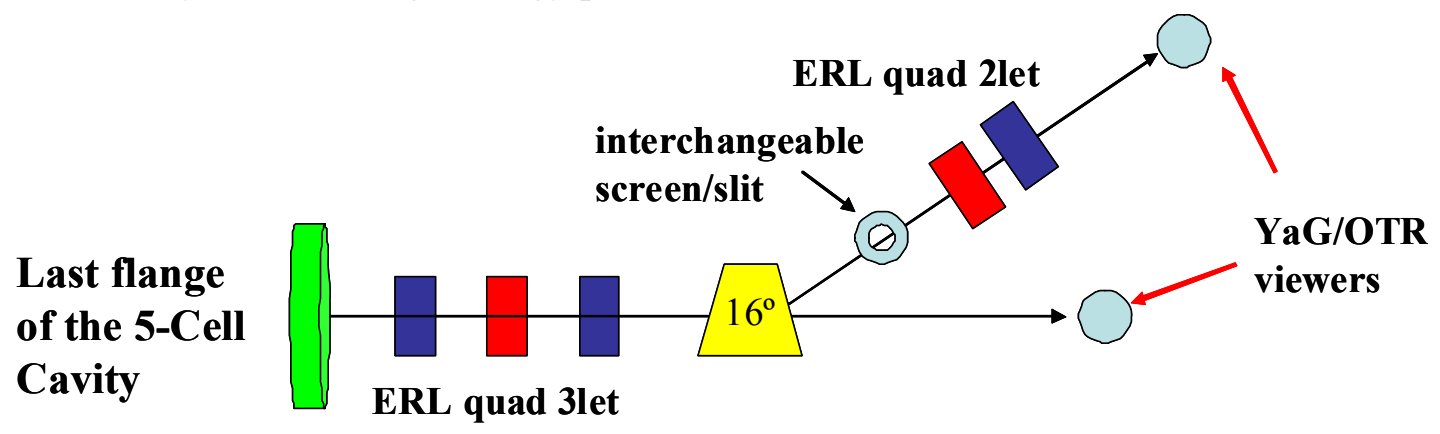

Figure 4: Layout of the high energy part of G5.

The setup has two operational modes:

- straight-ahead with the magnet off aimed at measuring

o projected emittance

0 transverse halo

- with the bend designed to measure

0 bunch length and longitudinal halo

o slice emittance.

\subsection{Straight-ahead path}

The program MADX was used to develop the optics of the high energy part of G5. Although MADX is much faster than PARMELA and more convenient program for developing the optics, MADX simulations effectively include only a single energy while PARMELA simulations are inclusive. To validate MADX results, the optical functions were simulated with PARMELA. First, the triplet was tuned to focus the beam to a round spot with a minimum size on a screen at the end of the line using MADX. Using the same setting of the triplet, the beam dynamics was simulated with PARMELA, showing a behavior very similar to that predicted by MADX. Figure 5 clearly shows this similarity. The triplet gradient settings were $70.3,-117$, and $66.6 \mathrm{Gauss} / \mathrm{cm}$. for the first, second, and third quadrupoles respectively. The beam charge per bunch was $1.4 \mathrm{nC}$ ("beer-can") and the kinetic energy was $16.7 \mathrm{MeV}$. We note that the maximum designed gradient value for these quads is 400 Gauss $/ \mathrm{cm}$.

Each of the triplet quadrupoles individually can be used for a quadrupole scan with other two quads off. Figure 6 shows results of two simulated quadrupole scans performed with the second and third triplet quadrupoles (one quad at a time, all other quads are off). The figure shows the squared r.m.s beam size simulated with PARMELA as a function of the quadrupole gradient (points). If the finite length of the quads and the space charge can be 


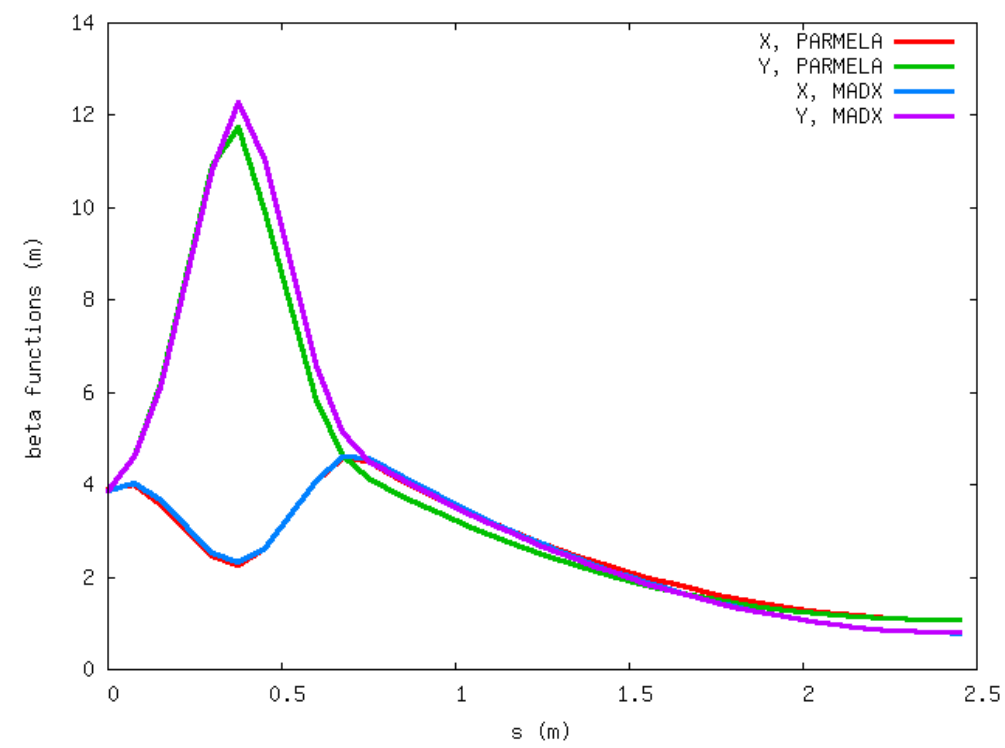

Figure 5: Beta functions in the straight-ahead line of the high energy section of G5 calculated by MADX and PARMELA. The initial values of the optical functions were simulated by PARMELA in a separate gun-to-cavity simulation run.

neglected, the data points have to form a parabola. Figure 6 shows parabolic fits to the simulated data. The fits were used to obtain the beam parameters at the quad. Namely, The emittance

The emittance number obtained from the fit and that known directly from PARMELA were compared to each other and showed a good agreement. The goal of this exercise was to demonstrate that

- 3-4\% energy spread of the distribution does not affect the measurement,

- space charge field does not affect the measurements,

- thin lens approximation can be used to fit the quadrupole scan data.

Figure 6 clearly demonstrates feasibility of using the outlined procedure to correctly determine the beam emittance. Errors such as the viewer resolution were not included in this exercise. 


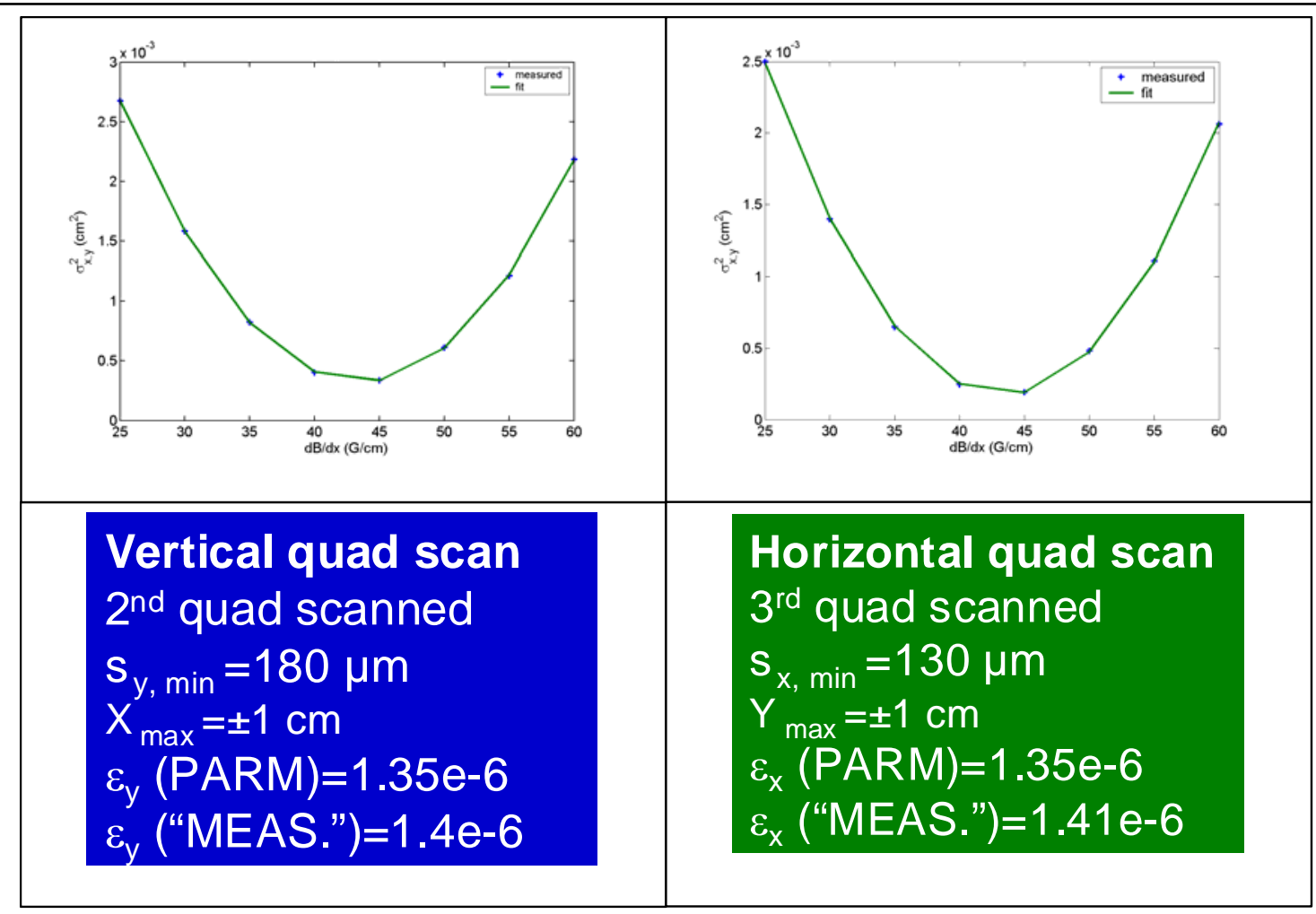

Figure 6: Quadrupole scans simulated with PARMELA (points) and fitted by parabolas (lines). Left: vertical quad scan by the second quad while other ones were off. Right: horizontal quad scan with the last quad. Colored boxes show other relavant parameters.

Also note that the last quadrupole of the triplet can be used to perform the quadrupole scan with other triplet quadrupoles set to their nominal values. PARMELA simulations show that this setup can be effectively used to measure the emittance. However, the error in reproducing the emittance number in this case was larger than that that in the case described above (only one quad was powered).

\subsection{The bend: energy spread and slice emittance measurements}

The simplest ways to measure the bunch length is to accelerate the beam off-crest, deflect the beam using a dipole magnet, and measure the bunch width in the deflection plane using a screen. A similar technique can be used to cut a longitudinal slice out of the beam if a narrow slit is used instead of the screen. Measuring the beam current going through the slit as a function of the phase shift, one can determine the longitudinal charge density distribution.

In G5 design, an ERL dipole, situated after the triplet and rotated by $30^{\circ}$, is used to deflect the beam and produce the dispersion function. The nominal deflection angle is $16^{\circ}$. In this setup, the entrance and exit angles are $-14^{\circ}$ and $2^{\circ}$ degrees respectively. To obtain a good energy-phase resolution, the horizontal betatron size has to be small at the location of the screen/slit. Figure 7 shows the beta-functions starting before the triplet and 
ending at the location of the energy resolution slit/screen for three values of the triplet strength: the nominal strength, $90 \%$ and $110 \%$ of the nominal strength. In all the cases, the horizontal beta function stays below $1 \mathrm{~m}$ at the location of the slit. Figure 8 shows the horizontal distribution of a bunch vs. RF phase at the location of the slit for on-crest acceleration and for a bunch shifted by $20^{\circ}$ off-crest. This figure clearly demonstrates that a $1 \mathrm{~mm}$ slit provides a longitudinal resolution of a few degrees for the shifted bunch.
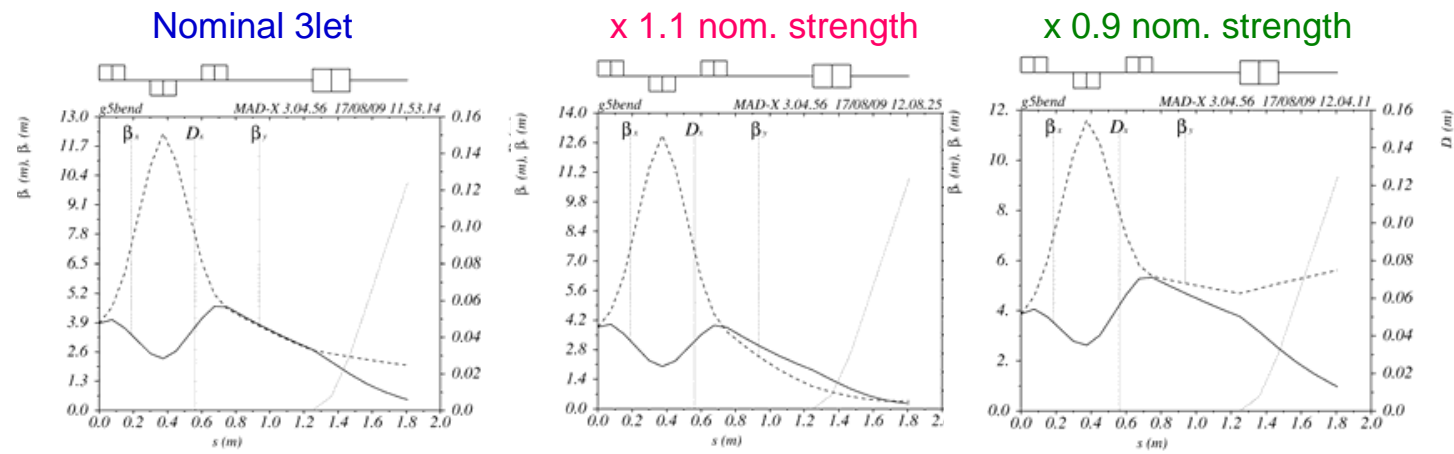

Figure 7: Beta-functions for three different triplet strengths. The initial conditions are the same for all the cases. The horizontal beta-function is always strongly focused by the triplet/dipole system, providing a good energy-phase resolution even for off-crest bunches.

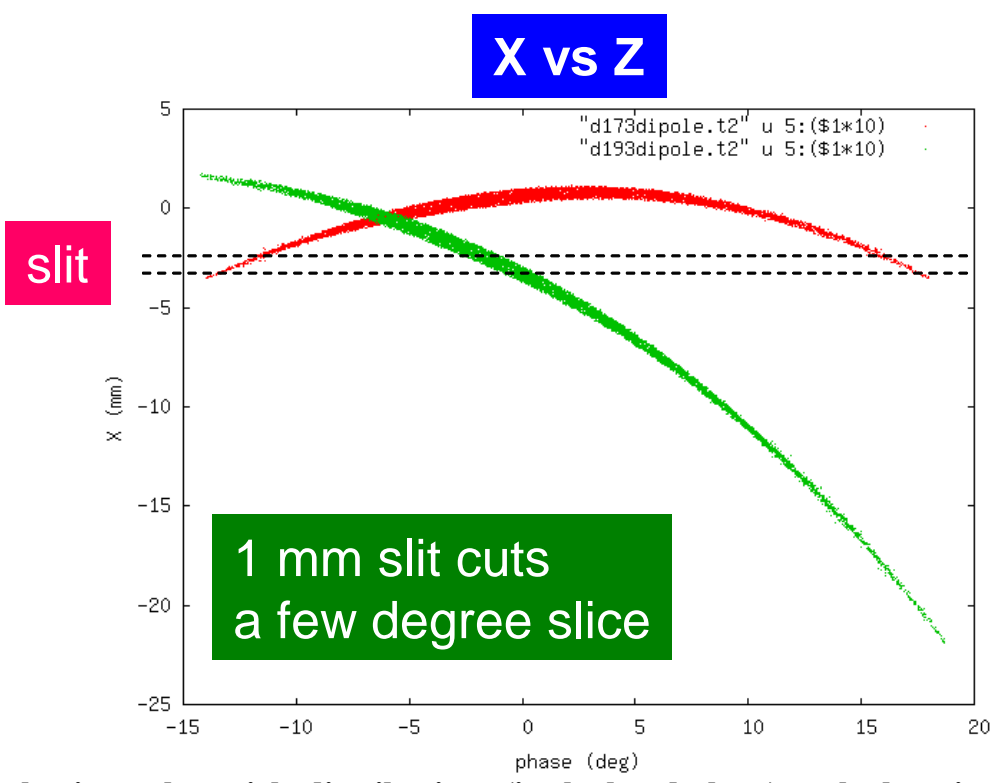

Figure 8: Two horizontal particle distributions (in the bend plane) at the location of the slit as a

function of the RF phase. The distributions are given for the on-crest and $20^{\circ}$ off-crest acceleration cases. $1 \mathrm{~mm}$ slit is capable of cutting out a-few-degree-long longitudinal slices out of the bunch shifted off-crest by $20^{\circ}$.

\subsubsection{Slice parameters of the off-crested beam}

To cut a specific slice the beam has to be accelerated off-crest as discussed above. Accelerating at a different phase affects the phase space of the beam due to somewhat different RF focusing. To estimate the effect, we calculated slice parameters (emittance 
and $\alpha$ - and $\beta$-functions) of a bunch accelerated on-crest and one accelerated $20^{\circ}$ off-crest. These bunch distributions were tracked by PARMELA from the gun to the end of the 5cell cavity cryomodule. Figure 9 shows that bunches accelerated on-crest and off-crest have almost identical sliced emittances. Slice $\alpha$ - and $\beta$-functions are shown in Figure 10. They also show small difference.

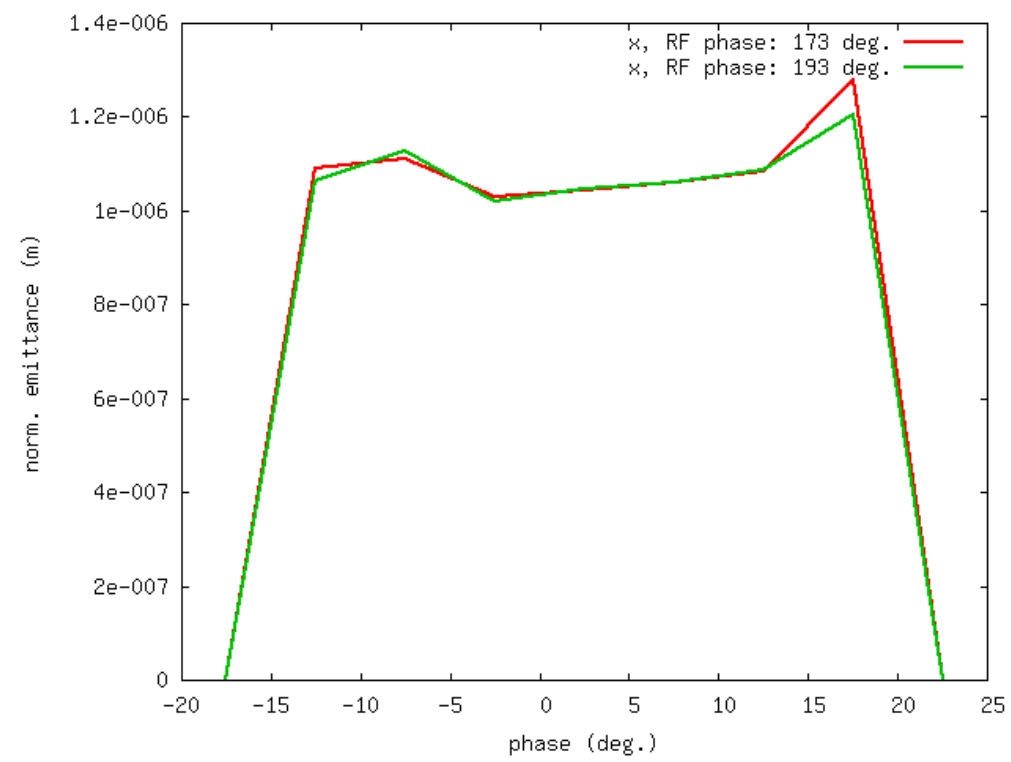

Figure 9: Slice emittance of bunches accelerated on-crest $\left(173^{\circ}\right)$ and $20^{\circ}$ off-crest $\left(193^{\circ}\right)$.

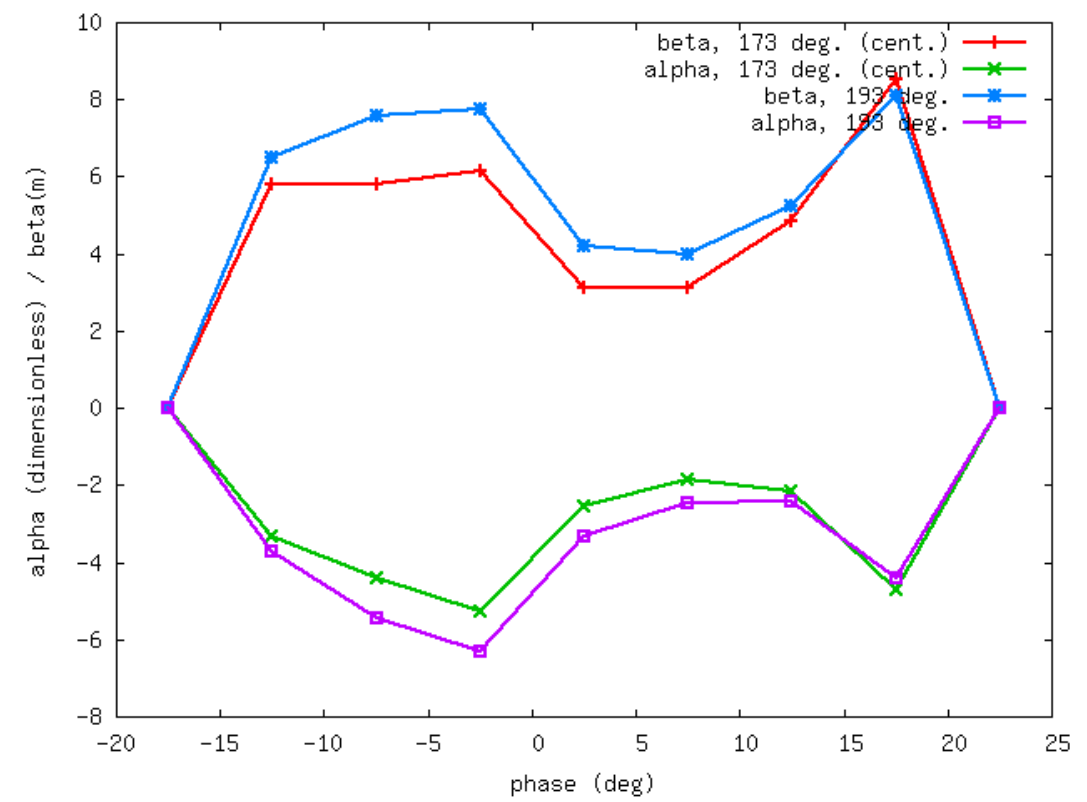

Figure 10: $\alpha$ - and $\beta$-functions of bunches accelerated on-crest $\left(173^{\circ}\right)$ and off-crest $\left(193^{\circ}\right)$. 


\subsubsection{Tuning the $\mathbf{G} 5$ high energy part}

Currently, the easiest way to tune the high energy parts appears to be as described below:

- Tune the straight-ahead path: minimize both $\mathrm{x}$ and $\mathrm{y}$ beam sizes and make a round beam spot at the viewer at the end of the straight ahead line. Figure 11.LEFT shows the beta-functions for this case.

- Turn on the dipole. The beam will be deflected towards the slit/screen located between the dipole and the doublet. The horizontal beta-function is not valid after the slit because while the vertical is correct. Figure 10.MIDDLE shows the optical functions in this case.

- Increase the last quadrupole of the triplet by approx. $10 \%$. Scan the first quadrupole of the doublet to do the quadrupole scan. This is shown in figure 10.RIGHT.

- It important to note that this procedure was still applicable without changes even if the initial $\beta$ - and $\alpha$-functions were 3 times larger than those used for the displayed example.
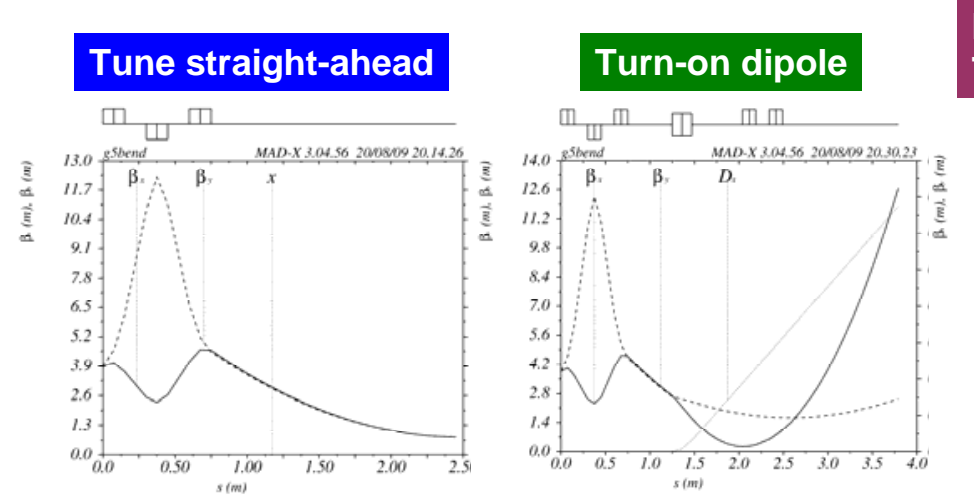

Increase $3^{\text {rd }}$ 3let quad $+10 \%$ Turn on $1^{\text {st }} 2$ let quad

Figure 1: Three tuning step of G5 high energy part. Left: tune the straight ahead part. Middle: turn on the dipole magnet. Right: increase the $3^{\text {rd }}$ quadrupole of the triplet and scan the first quad of the doublet to perform the quadrupole scan.

\section{Commissioning Plan}

We developed the straight pass (gun -- 5 cell cavity -- beam stop) test for the SRF Gun performance studies.

1) We will start commissioning with metal cathode (extremely low charge per bunch).

2) Multi-Alkaline Cathode single bunch with 0.7-5 $\mathrm{nC}$ per bunch.

3) Will operate in train mode $10 \mathrm{MHz}$ bunches in the train with:

» max duty factor $2 \mathrm{E}-5$.

" maximum average current $1 \mu \mathrm{A}$,

" max average power to the beam dump $20 \mathrm{~W}$

4) Replace straight line to z-bend to study the emittance conservation in a novel merger system: Zigzag merger.

5) Install the rest of the ERL return loop and demonstrate energy recovery at 50 $\mathrm{mA}$. 
6) After recirculation loop completed, demonstrate energy recovery of high charge and high current beam. The prototype will serve as a test bed for studying issues relevant for very high current ERLs 